fene schämen wir uns für die Verantwortlichen, schämen wir uns an ihrer Stelle dafür, welches Bild Deutschland mit diesem „Nein“ im europäischen Kontext abgibt. Da tut es gut, zu wissen, dass es auch in der Bundesregierung andere Stimmen gibt, Frauen und Männer, die sich für die gleichberechtigte Teilhabe von Frauen in allen gesellschaftlichen Bereichen ein- setzen und unsere Forderung nach einer Quote jedenfalls für die Aufsichtsräte in der Privatwirtschaft unterstützen.

Ich bin gespannt auf die Diskussion mit Ihnen und freue mich auf Ihre Erfahrungen und den Austausch.

Haben Sie ganz herzlichen Dank für Ihre Aufmerksamkeit.

\title{
Ende der Märchenstunde - Über das Scheitern der Frauenpolitik in der 17. Legislaturperiode
}

\section{Katrin Lange}

Projektmitarbeiterin, Deutscher Juristinnenbund, Berlin

Am 18. April 2013 stand auf der Tagesordnung der Plenarsitzung des Deutschen Bundestages die Abstimmung über gleich mehrere Initiativen zur Einführung einer Quote in Führungsgremien. ${ }^{1}$ Nicht dass mit einer Überraschung im Abstimmungsverhalten der gewählten Vertreterinnen und Vertreter dieser Republik wirklich zu rechnen gewesen wäre, wenige Tage vor der Debatte stand spätestens die bevorstehende Ablehnung der Gesetzesanträge fest. Die Haltung der Union konnte, trotz Zugeständnissen der Opposition ${ }^{2}$, in Hinblick auf eine Zustimmung der Initiativen nicht beeinflusst werden. Um aber einem drohenden Abstimmungsdilemma zu entgehen, hatte die CDU in einer bemerkenswerten Kehrtwende ihre Reihen mit einem Kompromiss geschlossen: die Aufnahme einer festen Quote von 30 Prozent im Jahr 2020 ins anstehende Wahlprogramm. In der Folge stimmten selbst Befürworter/innen der Quote gegen die Anträge. Eine notwendige Mehrheit konnte erwartungsgemäß somit nicht erreicht werden.

Was ist nun der Stand der Dinge? Trotz des erneut gescheiterten Versuchs, eine Quote für Führungsgremien gesetzlich zu verankern, und der somit historisch verpassten Chance, gleichberechtigte Teilhabe von Frauen in der Privatwirtschaft zu sichern, bleibt der Druck auf Politik und Wirtschaft ungebrochen.

In der Berliner Erklärung ${ }^{3}$ kämpfen seit Dezember 2011 Vertreterinnen führender Frauenverbände ${ }^{4}$ und Abgeordnete aller sechs im Bundestag vertretenen Parteien gemeinsam für eine Quote von mindestens 30 Prozent Frauen für die Aufsichtsräte. Diese Allianz, überfraktionell und zwischen Politik und Zivilgesellschaft geschlossen, ist einzigartig und wird mittlerweile von knapp 23.000 Unterzeichnerinnen und Unterzeichnern mit getragen. Ausdrücklich wird sich so zu dem gemeinsamen Ziel, Frauen und Männern in allen gesellschaftlichen Bereichen gleiche Verwirklichungs- und Teilhaberechte einzuräumen, bekannt. Dies zeigt auch, welche Bedeutung die Debatte über eine gesetzliche Regelung einer Frauenquote in der Gesellschaft inzwischen eingenommen hat. In einem kurz vor der Abstimmung im Bundestag veröffentlichten offenen Brief forderten die Initiatorenverbände der Berliner Erklärung zusammen mit vier weiteren Verbänden ${ }^{5}$ alle 620 Abgeordneten des Bundestages ausdrücklich auf, für die Initiative des Bundesrats, die zur namentlichen Abstimmung stand, zu stimmen. Im Ergebnis stimmten von 598 Abgeordneten 320 mit Nein und 277 für Ja. Es gab eine Enthaltung. ${ }^{6}$ Einzig Sibylle Laurischk, Abgeordnete der FDP-Fraktion und MitInitiatorin der Berliner Erklärung, stimmte zusammen mit der Opposition für den Gesetzesantrag. ${ }^{7}$

Die CDU hat mit ihrem Nein zur Quote die letzte Möglichkeit verstreichen lassen, noch in dieser Legislaturperiode einen deutlichen Impuls hinsichtlich des Stellenwerts von Frauen in Wirtschaft und Gesellschaft zu setzen. Hervorzuheben bleibt allein, dass ihr Kompromiss einen seit Jahren andauernden parteiinternen Streit zwischen Bundesarbeitsministerin Ursula von der Leyen und Bundesfamilienministerin Kristina Schröder über die Ausgestaltung von Maßnahmen, die zur Erhöhung des Anteils von Frauen in Führungspositionen beitragen sollen, zu beenden scheint. Erstmals ist eine gesetzliche Festlegung einer festen Frauenquote für Unternehmen in der CDU überhaupt vorgesehen. Lange hat Ursula von der Leyen sich dafür eingesetzt. Letztlich half der Druck, den sie durch ihre bis dahin offen gelassene Überlegung auslöste, sich der Opposition anzuschließen und für die Quote zu stimmen.

Nun sind also beide Quotenkonzepte im Kompromiss vereint. Bis 2020 wird die parteiintern nicht unumstrittene FlexiQuote, die vorrangig auf freiwillige Selbstverpflichtung der Unternehmen setzt, verfolgt. Sollte sich kein nennenswerter Erfolg einstellen, kommt ab 2020 die feste Quote. Die Idee, auf freiwillige Selbstverpflichtungen der Wirtschaftsunternehmen zu setzen, konterkariert die Union somit in zweifacher Hinsicht. Zum einen, indem sie die Flexi-Quote selbst zum

1 Informationen zu den Gesetzesinitiativen in: Lange, Katrin, Was bisher geschah. Das Ringen um die gesetzliche Regelung einer Frauenquote in wirtschaftlichen Führungspositionen, in dieser Ausgabe S. 70.

2 Nicht nur, dass die Gesetzesinitiativen selbst Produkt eines Minimalkonsenses der verschiedenen Fraktionen sind, in einem am Vortag der Abstimmung eingereichten Änderungsantrag der Grünen wurde in allerletzter Sekunde auf den kurz zuvor geschlossenen Konsens in der Unionsfraktion eingegangen, demzufolge entsprechend für 2020 eine Quote von 30 Prozent Frauenanteil vorgesehen ist.

3 <http://www.berlinererklaerung.de/> (Zugriff: 18.4.2013).

4 Die insgesamt zehn Frauenverbände und -initiativen haben mehr als 500.000 Mitglieder und Unterstützer/innen.

5 Deutscher Ingenieurinnenbund e.V., ProQuote Medien e.V., ProQuote Medizin und Nürnberger Resolution.

6 Siegfried Kauder, Abgeordneter der CDU/CSU-Fraktion und Vorsitzender des Rechtsausschusses im Bundestag, Bruder von Volker Kauder, Vorsitzender der CDU/CSU-Fraktion.

7 <http://dip21.bundestag.de/dip21/btp/17/17234.pdf> (Zugriff: 19.4.2013) 
Auslaufmodell macht. Zum anderen, indem sie selbst bei der am 18. April 2013 hitzig geführten und von Zwischenrufen begleiteten Plenardebatte das Scheitern der 2001 beschlossenen Vereinbarung zur Förderung der Chancengleichbeit in der Privatwirtschaft zwischen der Bundesregierung und den Spitzenverbänden der deutschen Wirtschaft als Argument für das frühere Versagen der Opposition heranzieht. Die Erfahrung, dass reine Lippenbekenntnisse der Unternehmen keine Veränderung in Vorständen und Aufsichtsräten anstößt, wurde somit schon einmal und mehr als deutlich gemacht. Und obwohl die Union das Problem des fortwährenden Stillhalteabkommens zulasten der Frauen zumindest als probates Mittel zur Anklage der Opposition erkannt hat, setzt sie leider noch weitere sieben Jahre auf die Selbstregulierungskräfte des Marktes. Dennoch bleibt offen, ob über die bloße Absichtserklärung hinaus die Quote kommt. Mit dem Wunschkoalitionspartner FDP ist sie bisher nicht zu machen. Selbst die Flexi-Quote sollte ursprünglich in diesem Jahr gesetzlich festgeschrieben werden. Dieser Teil des Stufenplans wurde dann kurzerhand und mit bis heute ausbleibender Begründung auf die Zeit nach der anstehenden Wahl vertagt. Und Wahlprogramme sind weder Garant für eine Wiederwahl noch Garant für Gesetze. Das Thema Chancengleichheit muss zwingend auf der politischen Agenda bleiben. ${ }^{8}$ Den öffentlichen Druck hierfür können, wie die Märchenstunde im Parlament am 18. April 2013 gezeigt hat, nur die Aktivistinnen und Aktivisten in der Zivilgesellschaft herstellen.

8 S. auch: Wahlprüfsteine zur Bundestagswahl 2013 für das Arbeits-, Wirtschafts- und Gleichstellungsrecht (K1) in dieser Ausgabe, S. 55.

\section{djb-Initiative bringt mehr „Frauen in die Roten Roben“}

\section{Eva Schübel}

Vizepräsidentin des djb; Bundesanwältin beim BGH, Karlsruhe

Bei der Richterwahl am 21. März 2013 - der zweiten Runde nach Beginn unserer Initiative im Herbst 2011 - hat es wieder ein Auf und Ab der Emotionen gegeben. Jubel wegen des hervorragenden Ergebnisses für den Bundesgerichtshof (sechs von acht Stellen an Kandidatinnen) und Freude wegen einer Zweidrittelmehrheit beim Bundesarbeitsgericht - dagegen lange Gesichter im Übrigen: für den Bundesfinanzhof wurden zwei Richterinnen und fünf Richter und für das Bundessozialgericht eine Richterin und 4 Richter gewählt; das Bundesverwaltungsgericht ist wieder einmal Schlusslicht mit einem Männerduo. Aufs Ganze gerechnet liegt die Frauenquote bei 44 Prozent. Das sehr gute Ergebnis für den Bundesgerichtshof relativiert sich, wenn man berücksichtigt, dass im vergangenen Jahr zehn Richter und nur zwei Richterinnen gewählt worden sind. Rechnen wir 2012 und 2013 zusammen, ergibt dies für den Bundesgerichtshof 12 neue Richter und acht neue Richterinnen.

Wir müssen daher zu erreichen suchen, dass bei jeder künftigen Wahl für jedes Bundesgericht mindestens 50 Prozent Frauen gewählt werden. Anderenfalls wird der Anteil der Bundesrichterinnen erst in mehr als 20 Jahren hälftig sein, was inakzeptabel ist.

Besser sieht es bei den Wahlvorschlägen aus. Unsere Präsidentin hat jedes Treffen mit Präsidentinnen und Präsidenten der Oberlandesgerichte und der Bundesgerichte sowie den Leitungen der Justizministerien genutzt, das Gleichstellungsdefizit bei den obersten Bundesgerichten aufzuzeigen und unser Anliegen zu verdeutlichen. Zur Erinnerung: Bei der Wahl 2011 war die Zahl der weiblichen Kandidaten auf einem deprimierenden Tiefstand angelangt. Unter 23 Kandidaten für den Bundesgerichtshof befanden sich gerade drei Frauen sowie jeweils nur eine Frau unter 17 Bewerbern für das Bundesverwaltungsgericht und unter sieben Bewerbern für den Bundesfinanzhof. Dieser Befund veranlasste mich zu der
Initiative „Frauen in die Roten Roben“, die bereits 2012 Wirkung gezeigt hat. Es werden zunehmend mehr Frauen vorgeschlagen. Im letzten Jahr waren unter den Kandidaten für den Bundesgerichtshof ein Drittel Frauen. In diesem Jahr waren es sogar 19 Richterinnen von 37 Kandidaten (=51,5\%!). Das Schlusslicht nicht nur bei der Wahl selbst bildete das Bundesverwaltungsgericht mit einer Vorschlagsquote von nur 20 Prozent. Sehr erfreulich ist, dass überproportional viele weibliche Neuvorschläge Parteien zuzurechnen sind, die das Thema Gleichstellung erst seit Kurzem ernster nehmen. Gleiche Wahlchancen für Richterinnen bestehen freilich erst dann, wenn auf den Wahllisten für die einzelnen Bundesgerichte je zur Hälfte Frauen und Männer stehen.

Hauptsächlich bestücken die Länderjustizministerien bzw. diejenigen Ressorts, zu deren Geschäftsbereich der jeweilige Gerichtszweig gehört, die Wahllisten. Denn die im Richterwahlausschuss vertretenden Abgeordneten machen von ihrem Vorschlagsrecht eher zurückhaltend Gebrauch. Die Auswahl der Kandidatinnen und Kandidaten ist in den Ländern offensichtlich Chefsache. Jedenfalls werden die Stellen, die ein Bundesland bei den Bundesgerichten besetzen kann, selten bekannt gemacht oder gar ausgeschrieben. Die zuständigen Ministerien führen interne Listen und entscheiden in der Regel ohne die Beteiligung der Gleichstellungs- bzw. Frauenbeauftragten und Richtervertretungen, wer gelistet und schließlich für ein Bundesgericht vorgeschlagen wird. Ein durch und durch intransparentes Verfahren, das vor allem Interessenten mit guten Kontakten in die Hausleitungen der Ministerien und Oberlandesgerichte nützt. Hier gilt es anzusetzen.

Über das Netzwerk der Gleichstellungs- bzw. Frauenbeauftragten und -vertreterinnen der Landesjustizverwaltungen habe ich den Kontakt in die Länderjustiz gesucht. Dabei hat mir der Umstand, dass ich selbst die Gleichstellungsbeauftragte des Generalbundesanwalts bin, die Türen zu den Kolleginnen geöffnet. Dies erleichtert auch meine Teilnahme an NetzwerkTreffen, bei denen ich den Weg in die obersten Bundesgerich- 